\title{
Surface Characterizations of Sulfamerazine Polymorphs Using Atomic Force Microscopy, Transmission Light Microscopy and Raman Microspectroscopy
}

\author{
Xiaoping Cao, Changquan Sun, and Thomas J. Thamann
}

Pfizer Global Research and Development, 7000 Portage Road, Kalamazoo, MI 49001

Surface properties of drug crystals are very important for many pharmaceutical concerns and processes. Polymorphism, a common and important characteristic of pharmaceutical crystals, often significantly affects surface and solid-state properties such as density, habit, melting, mechanical properties and dissolution rates $[1,2]$. Therefore, it is of great value to study and understand surface properties of polymorphs. Recently, atomic force microscopy (AFM) has been shown to be a powerful tool to study crystals because it can not only characterize individual particles but also differentiate surface properties of polymorphs. In this study, AFM, transmission light microscopy and Raman microspectroscopy have been used to study Sulfamerazine (SMZ) single crystals.

SMZ is an antibacterial agent. Two polymorphs have been previously identified and characterized $[3,4]$. In this study, SMZ single crystals were prepared from aqueous dispersions of SMZ Form I and II bulk samples. Different crystal shapes were observed by the transmission light microscopy. Polymorphic nature of single crystals was identified by comparing peak positions of their Raman spectra with those of bulk samples, as shown in Fig. 1. The results suggested that rectangular single crystals were Form I while hexagonal ones be Form II. Furthermore, Raman microspectroscopy provided information on surface functional groups.

The surface morphology of individual single crystals was investigated by AFM with contact and tapping modes. Topographic images revealed a smooth surface, a uniform height and sharp edges for both Form I and II single crystals, as shown in Fig. 2. Detailed surface features of the single crystals were showed by topographic and phase images at a scanning scale of 1 micrometer. Crystalline terraces were observed for Form I but not for Form II, as shown in Fig. 3. Surface properties of single crystals were evaluated using AFM force measurements. Larger adhesion forces observed for the surface of Form I single crystals using hydrophilic silicon nitride tips suggested that the surface of Form I single crystal surface was more hydrophilic than that of Form II.

Theoretical calculations predicted that Form I had the (020) face dominant and Form II had the (002) face dominant. The predictions were consistent with Raman and AFM results.

References

[1] Grant, D. J. W. Polymorphism in Pharmaceutical Solids. In Theory and Origin of Polymorphism; Brittain, H. G., Ed.; Marcel Dekker, Inc.: New York, 1999; pp 1-34.

[2] Sun, C.; Grant, D. J. W. Pharmaceutical Research 18 (2001), 274-280.

[3] Caira, M. R.; Mohamed, R. Acta Cryst. B48 (1992), 492-498.

[4] Acharya, K. R.; Kuchela, K. N.; Kartha, G. J. Crystallorgr.Spectrosc. Res. 12 (1982), 369-376.

[5] The aid of Gary Nichols in theoretical calculations is gratefully acknowledged. 

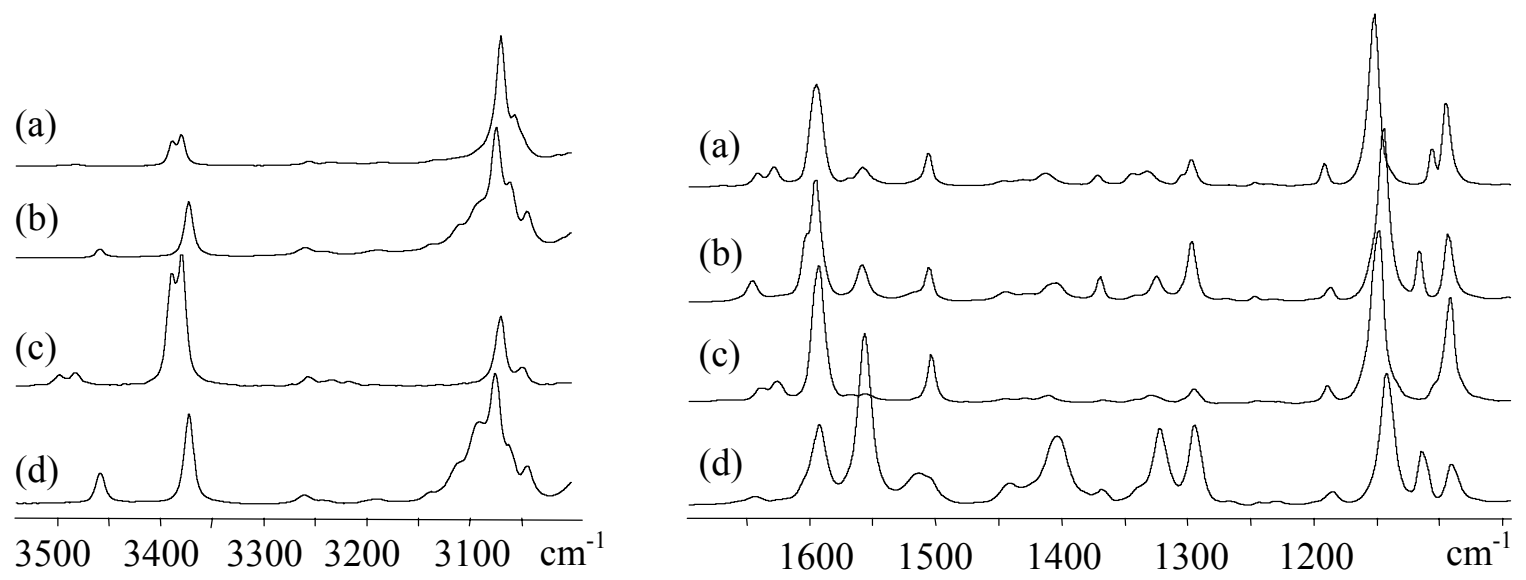

Fig. 1. Raman spectra of SMZ samples: (a) Form I bulk, (b) Form II bulk, (c) rectangle-shaped single crystal, and (d) hexagon-shaped single crystal. Raman spectra have identified the rectangleshaped single crystals are Form I and the hexagon-shaped ones are Form II.
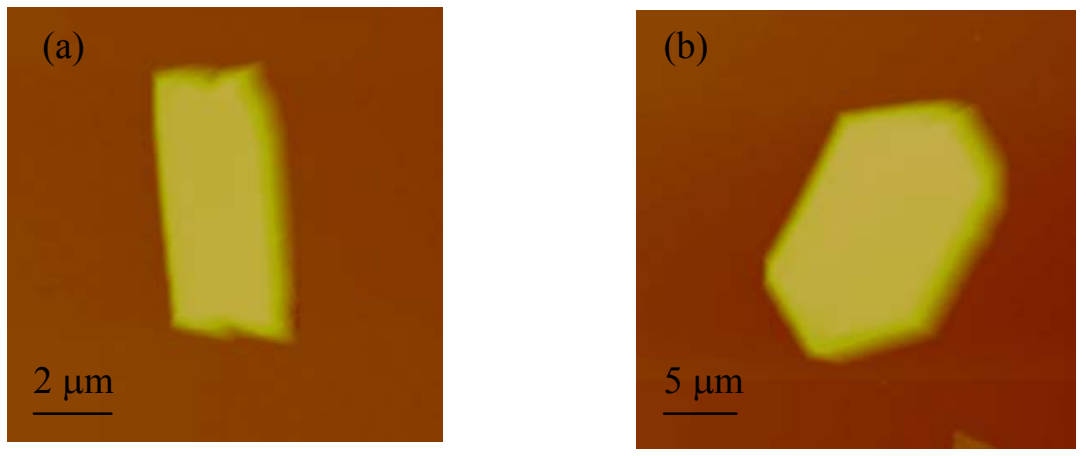

Fig. 2. Topographic images of SMZ single crystals: (a) Form I, (b) Form II. The images were acquired with a contact mode, showing different crystal shapes.
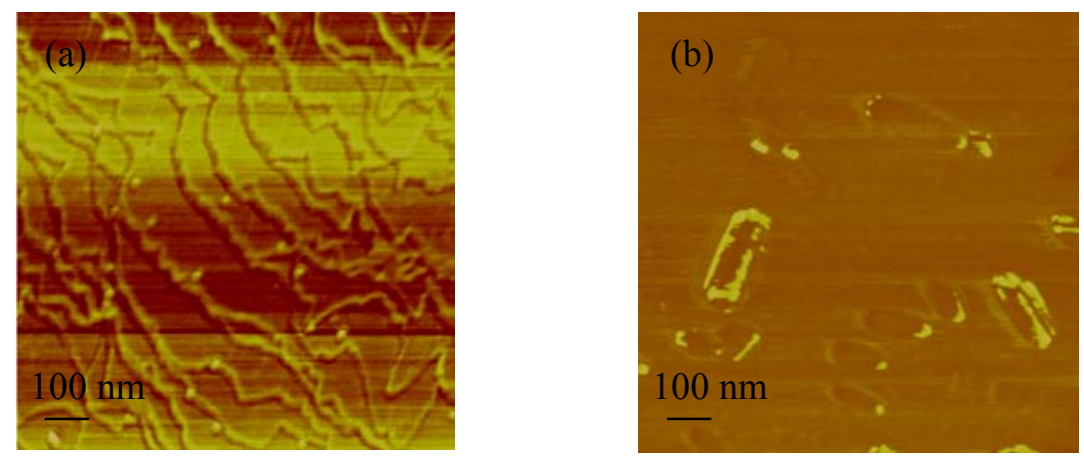

Fig. 3. Phase images of SMZ single crystals at a scanning scale of $1 \mu \mathrm{m}$ : (a) Form I, (b) Form II. Crystalline terraces with different step heights were observed for the Form I, while a relatively smooth surface with small protrusions was observed for the Form II. 